\title{
ANAESTHESIA FOR PERCUTANEOUS SPLENOPORTOGRAPHY IN CHILDREN
}

\author{
Code Smith, M.D., F.R.C.P.(c)*
}

PERCUTANEOLs splenoportography refers to a radiological investigation in which serial radiographs are taken in order to visualize the course of radio-opaque material injected into the splenic pulp, through the portal system of veins. An indication of portal venous pressure may be obtained by recording the intrasplenic pressure immediately preceding the injection of the contrast medium. In the series here presented this combined procedure was used.

Splenoportography is performed to determine the site of portal obstruction and the degree of portal hypertension. Additional useful information obtained from the procedure is the demonstration of oesophageal and/or gastric varices, which occur in some cases.

During the past seven years 29 such investigations have been performed on 22 patients at the Hospital for Sick Children and the Toronto General Hospital. Of these, 20 cases ( 27 procedures) were anaesthetized and injected by the same physician-anaesthetist team.

When first investigated, two patients were under one year of age; seven were between one and five years; four were between five and ten years; and the remaining seven patients were between ten and fourteen years of age.

\section{Degree of Hepatic Dysfunction}

Patients presenting with signs of portal obstruction may be divided into three groups. The obstruction may be prehepatic, usually on the basis of a congenital anomaly, or intrahepatic due to various types of cirrhosis. Patients in the first group have essentially normal liver function and constitute group I ( 9 cases, 12 procedures). The cirrhotics, none of whom were in a decompensated state, may be divided into mild cirrhotics, group II (5 cases, 5 procedures), with signs of minimal hepatic dysfunction, and moderately advanced cirrhotics, group III ( 8 cases, 10 procedures), with signs of severe liver dysfunction. In group $I$, three patients underwent two investigations; in group III two patients underwent two investigations; and two patients were classed in group II for their first, and in group III for their second investigation.

\section{Procedure}

The patient is positioned on the X-r thy table; the spleen is palpated and outlined in ink; the abdomen is prepared and draped. Anaesthesia is then induced (details are given below). A No. 20 gauge needle is inserted into the spleen and a pressure is recorded. The contrast material is then rapidly injected under a high pressure and serial radiographs are taken. The needle is withdrawn and a dressing applied. The patient is turned into the left lateral position and a pillow is placed

\footnotetext{
*Associate, Department of Anaesthesia, University of Toronto, Toronto, Ontario.
} 
under him at the level of the puncture. This position is maintained during recovery and for a period of two hours after anaesthesia. A q.1. h. chart is kept for the first hour after anaesthesia, and a $q \cdot \frac{1}{2} . h$. chart for the second hour. Thereafter the pulse rate and blood pressure are recorded hourly for eight hours.

\section{Anaesthetic Requirements ${ }^{1}$}

Three factors are of importance in anaesthetizing these patients:

1. A non-explosive technique because of the proximity to X-ray and electrically operated measuring devices.

2. Complete apnoea throughout the investigation. The splenic capsule is easily torn if the spleen is allowed to move up and down against a needle fixed at its insertion through the abdominal wall.

3. Minimal doses of anaesthetic drugs. Patients with impaired liver function should receive minimal amounts of narcotics, barbiturates, and relaxants.

\section{Anaesthetic Technique}

Premedication with narcotics was avoided in this series. One highly nervous lad in group I received $0.75 \mathrm{mg}$./1b. meperidine; however, all other cases received only atropine sulphate, usually intravenously, with the barbiturate induction.

It is natural that the technique employed over a period of seven years should evolve, and there have been several phases during this period. Each phase is marked by a variation in the type or dosage of inducing drug and relaxant. In every case hyperventilation with 100 per cent oxygen was begun immediately following the intravenous injections and continued for a full minute following the onset of apnoea. There was no manipulation of the anaesthetic bag during the 45 to 60 second period during which the tests were performed. The patients were gently ventilated from the time the needle was removed until adequate respiration was resumed.

The first six cases received thiopentone $(3 \mathrm{mg} . / \mathrm{lb}$.) and decamethonium. During this phase the procedure was a novel one for the team and adequate time for the procedure was achieved through the use of a longer-acting relaxant. On several occasions a splenic pressure could not be obtained and the needle had to be' reinserted, and other technical problems with the recording apparatus were encountered. With experience the dose of decamethonium was reduced from 1 $\mathrm{mg} . / 50 \mathrm{lb}$. to $1 \mathrm{mg} . / 100 \mathrm{lb}$., and $1 \mathrm{mg} . / 4 \mathrm{lb}$. succinylcholine was added.

The next seven cases received no decamethonium. They were given $2-3 \mathrm{mg}$./ $/ \mathrm{b}$. thiopentone and $1 \mathrm{mg} . / 3.5 \mathrm{lb}$. succinylcholine, a reduction in dosage in keeping with a more smoothly performed investigation.

At this time methitural sodium was introduced and the next seven cases received this drug in doses of $4 \mathrm{mg}$./ $/ \mathrm{b}$. and succinylcholine. These cases have been reported previously. ${ }^{2}$

The last seven cases received $1.5-2.0 \mathrm{mg}$./ $/ \mathrm{lb}$.: thiopentone and $1 \mathrm{mg} . / 4 \mathrm{lb}$. succinylcholine. This combination provided just enough time for the completion of the investigation and resulted in very rapid return of both respiration and consciousness.

It will be seen that as a result of practice and co-operation between the members 
comprising the team the pharmacological insult to these cases has been gradually reduced. Those unfamiliar with the procedure might well adopt a somewhat similar approach to the handling of these patients.

\section{COMPLICATIONS}

1. Delayed recovery. Prolonged apnoea did not occur in this series. However, two patients in group III who had received methitural sodium and succinylcholine regained consciousness before respiration was considered adequate. They were assisted with a mixture of 80 per cent nitrous oxide and 20 per cent oxygen for about five minutes, and subsequently had no recollection of waking up and being unable to breathe.

2. Intraperitoneal haemorrhage. None of these cases showed signs of shock or intraperitoneal irritation and bleeding. We feel that this is due to the complete apnoea during the time that the needle is within the splenic pulp.

\section{Summary}

Percutaneous splenoportography can be performed in less than one minute. It is essential that complete apnoea should be present during this time. Any non-explosive technique may be employed when dealing with patients with normal or nearly normal hepatic function. A reduction in the pharmacological insult to those with abnormal function can be obtained by pre-anaesthetic preparation and draping and the use of minimal amounts of hypnotic and shortacting relaxant.

\section{ACKNOWLEDGMENT}

The author wishes to acknowledge the advice and assistance of Dr. Andrew Sass-Kortsak who performed the investigations herein described.

\section{REFERENCES}

1. Bowen, R. A. Anaesthesia in Operations for the Relief of Portal Hypertension. Anaesthesia - 15: 3-10 (1960).

2.Smith, Code. Methitural Sodium for the Induction of Paediatric Anaesthesia. Canad Anaesth. Soc. J. 4: 378-383 (1957). 